\title{
The Generation of Random Numbers That Are Probably Prime
}

\author{
Pierre Beauchemin and Gilles Brassard ${ }^{1}$ \\ Département d'informatique et de recherche opérationnelle, Université de Montréal, \\ C.P. 6128, Succ. "A", Montréal, Québec, Canada H3C3J7 \\ Claude Crépeau ${ }^{2}$ \\ Massachusetts Institute of Technology, Department of Computer Science, \\ 545 Technology Square, Cambridge, MA 02139, U.S.A.
}

\section{Claude Goutier}

Centre de Calcul, Université de Montréal, C.P. 6128, Succ. "A", Montréal, Québec, Canada H3C3J7

\section{Carl Pomerance ${ }^{3}$}

Department of Mathematics, University of Georgia, Athens, GA 30602, U.S.A.

\begin{abstract}
In this paper we make two observations on Rabin's probabilistic primality test. The first is a provocative reason why Rabin's test is so good. It turned out that a single iteration has a nonnegligible probability of failing only on composite numbers that can actually be split in expected polynomial time. Therefore, factoring would be easy if Rabin's test systematically failed with a $25 \%$ probability on each composite integer (which, of course, it does not). The second observation is more fundamental because it is not restricted to primality testing: it has consequences for the entire field of probabilistic algorithms. The failure probability when using a probabilistic algorithm for the purpose of testing some property is compared with that when using it for the purpose of obtaining a random element hopefully having this property. More specifically, we investigate the question of how reliable Rabin's test is when used to generate a random integer that is probably prime, rather than to test a specific integer for primality.
\end{abstract}

Key words. Factorization, False witnesses, Primality testing, Probabilistic algorithms, Rabin's test.

\section{A Brief Survey of Primality Testing}

How difficult is it to distinguish prime numbers from composite numbers? This is perhaps the single most important problem in computational number theory. We

\footnotetext{
${ }^{1}$ Supported in part by NSERC grant A4107. Part of the research was performed while this author was at the CWI, Amsterdam.

${ }^{2}$ Supported in part by an NSERC Posgraduate Scholarship. Part of the research was performed while this author was at the Université de Montréal.

${ }^{3}$ Supported in part by an NSF grant.
} 
do not attempt here an exhaustive review of its long history. Let us only mention some of the most outstanding modern steps. It has been known for several years that the problem of recognizing prime numbers belongs to $\mathbf{P}$ under the Extended Riemann Hypothesis [15] and that it belongs to Co-RP [20], [22] and NP [19] without any assumptions. It can also be solved in almost polynomial time by a deterministic algorithm that runs for a number of steps in $O\left(m^{O(\log \log m)}\right)$ [2], [17], [7], where $m$ is the size of the number to be tested, that is the number of bits in its binary representation. More recently, it was found to lie in RP [11], [1], and therefore in ZPP [10] as well. In other words, this problem can be solved in probabilistic polynomial time by a Las Vegas [3] algorithm: whenever an answer is obtained, that answer is correct.

From a theoretical point of view, the problem of primality of primality testing is therefore essentially solved: the only remaining question is to figure out whether or not it belongs to $\mathbf{P}$ without assumptions. However, the polynomial that gives the running time of [11] is of the twelfth degree and [1] only makes things worse in practice. Despite Atkin's much more reasonable version of these algorithms, Rabin's probabilistic test [20] remains the best approach for very large numbers (several hundreds of decimal digits). Let $\operatorname{prob}[\operatorname{Rabin}(n)=$ verdict $]$ denote the probability that one iteration of this algorithm on input $n$ returns verdict, where verdict can either be "prime" or "composite." The basic theorem about Rabin's test is that

$$
\operatorname{prob}[\operatorname{Rabin}(n)=" \operatorname{prime} " \mid n \text { is indeed prime }]=1
$$

whereas

$$
\operatorname{prob}[\operatorname{Rabin}(n)=\text { "prime" } \mid n \text { is in fact composite }] \leq 1 / 4 \text {. }
$$

One is therefore certain that $n$ is composite whenever any single run of Rabin(n) returns "composite." On the other hand, one can never be sure that $n$ is prime no matter how many runs of Rabin(n) have returned "prime." This test is usually run in a loop as follows:

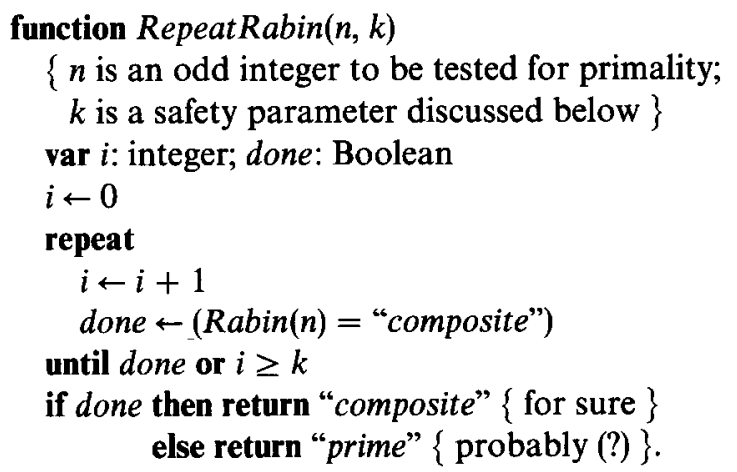

There is a tradeoff in the choice of the parameter $k$ above: the bigger it is, the more confident we are in the advent of a "prime" answer but the more time it takes to build up this confidence. This paper addresses two aspects of the question: just how confident in a number's primality can we be after running this test? 


\section{Rabin's Test in Relation to Factoring}

Let $n$ be an odd integer, $n>1$, and let $v$ and $u$ be integers such that $n-1=2^{v} u$, where $u$ is odd. Define

$$
\mathbb{Z}_{n}^{*}=\{a \mid 1 \leq a<n \text { and } \operatorname{gcd}(a, n)=1\}
$$

and

$$
\mathrm{R}_{n}=\left\{a \in \mathbb{Z}_{n}^{*} \mid a^{u} \equiv 1(\bmod n) \text { or }(\exists j)\left[0 \leq j<v \text { and } a^{2^{j u}} \equiv-1(\bmod n)\right]\right\} .
$$

The basic theorem about Rabin's test states that $\mathrm{R}_{n}=\mathbb{Z}_{n}^{*}$ whenever $n$ is a prime, whereas $\# \mathrm{R}_{n} \leq \# \mathbb{Z}_{n}^{*} / 4$ otherwise, where $\# X$ denotes the number of elements in set $X$. Notice that both 1 and $n-1$ always belong to $\mathrm{R}_{n}$. This theorem is normally used as follows:

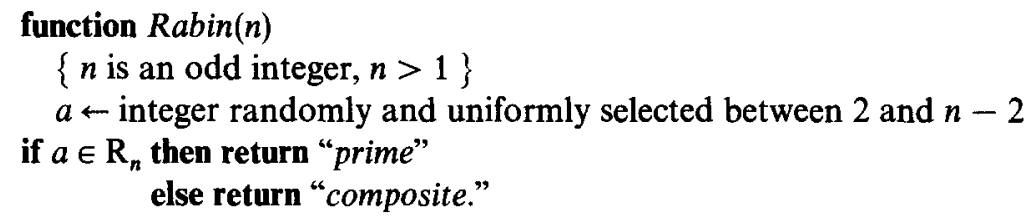

Whenever $n$ is composite, the error probability of this procedure is clearly given by $\left(\# \mathrm{R}_{n}-2\right) /(n-3)$, so that elements of $\mathrm{R}_{n}$ others than 1 and $n-1$ are known as Rabin false witnesses for $n$. From the basic theorem we know that this error probability is always smaller than $25 \%$. However, it is well known to be often much smaller. Monier gives an exact formula for this probability [16]; see also [14]. As a corollary of Monier's formula, the error probability never exceeds $\left(\varphi(n) / 2^{r-1}-2\right) /(n-3)$, where $r$ is the number of distinct prime factors of $n$ and $\varphi(n)=\# \mathbb{Z}_{n}^{*}$ denotes Euler's function [12]. Despite this tightening of the bound on the error probability (at least when $n$ has more than three distinct prime factors), it turns out that the latter is usually still much smaller. In other words, Rabin's test performs in practice much better than one might naively expect.

For instance, $42,799(=127 \times 337)$ admits only 880 Rabin false witnesses, compared with $\varphi(42,799) / 4=10,584$. Even better, Rabin's test never fails on integers of the form $3 \times 5 \times 7 \times 11 \times \cdots$ such as 15,015: these admit no false witnesses at all. More impressively, it is enough to test deterministically for each $a \in\{2,5,7,13\}$ in order to decide primality without any failures up to $25 \times 10^{9}$ (using $\{2,3,5,7\}$ still leaves one error in this range [18]). Although "high risk" numbers exist, such as $n=79,003(=199 \times 397)$ or $3,215,031,751(=151 \times 751 \times 28,351)$ with $\# \mathrm{R}_{n}=\# \mathbb{Z}_{n}^{*} / 4$ and $\left(\# \mathrm{R}_{n}-2\right) /(n-3) \approx 24.8 \%$, these are not the rule. (One can nonetheless prove, using Monier's formula, that every composite number of the form $(12 m+7)(24 m+13)$ is such a high-risk number, provided both $12 m+7$ and $24 m+13$ are prime-but the existence of infinitely many such numbers remains an open question.) We now show that it is somewhat unfortunate (except for cryptographers!) that Rabin's test is so good, because otherwise factoring would be easy.

For this purpose, consider the set of Fermat false witnesses for $n$ :

$$
F_{n}=\left\{a \in \mathbb{Z}_{n}^{*} \mid a^{n-1} \equiv 1(\bmod n)\right\}
$$


Obviously, $\mathrm{R}_{n} \subseteq \mathrm{F}_{n}$ for each odd integer $n>1$. Define $\mathrm{H}_{n}=\mathrm{F}_{n} \backslash \mathrm{R}_{n}$, the set of Fermat false witnesses that are however not Rabin false witnesses. Assume for the moment that $n$ is not a prime power (i.e., not of the form $p^{m}$ for some prime $p$ and some integer $m \geq 1$ ). Theorem 1 states that each element of $\mathrm{H}_{n}$ is a handle that allows easy splitting of $n$ (i.e., finding at least one nontrivial factor of $n$ ) and that there are at least as many such handles as there are Rabin false witnesses. Our provocative interpretation states that it is only possible for even a single iteration of Rabin's test to fail (i.e., declare $n$ prime) with a nonnegligible probability if it happens that $n$ is easy to split (and hence obviously composite)! This result extends to every composite $n$ since even numbers and prime powers are easy to split. In other words, there exists a simple probabilistic splitting algorithm whose running time is small on every composite number on which Rabin's test is not extremely effective. More precisely, for any function $t(l)$, our splitting algorithm succeeds at finding a nontrivial factor within expected time in the order of $l^{3} t(l)$ on every $l$-digit composite integer $n$ such that $\operatorname{prob}[\operatorname{Rabin}(n)=$ "prime" $] \geq 1 / t(l)$. In particular, it runs in expected polynomial time on these integers if $t(l)$ is bounded by some fixed polynomial.

Another consequence of this result is that whenever Fermat's test fails to recognize a composite integer as such whereas Rabin's test would not have failed (with the same random choices), this means that Fermat's test has just missed a golden opportunity to split the given integer! (This phenomenon has already been observed by Baillie and Wagstaff $[4$, p. 1402].)

Theorem 1. Let $n$ be any odd composite integer that is not a prime power, and let $v$ and $u$ be integers such that $n-1=2^{v} u$, where $u$ is odd:

(i) $\left(\forall a \in \mathrm{H}_{n}\right)(\exists j<v)\left[\operatorname{gcd}\left(n, a^{2^{j} u}+1\right)\right.$ is a nontrivial factor of $\left.n\right]$.

(ii) $\# \mathrm{H}_{n} \geq \# \mathrm{R}_{n}$.

Proof. (i) Consider any $a \in \mathrm{H}_{n}$. Let $i$ be the smallest integer such that $a^{2^{i} u} \equiv$ $1(\bmod n)$. We have $0 \leq i \leq v$ because $a \in \mathrm{F}_{n}$. However, $i=0$ is not possible since $a \notin \mathbf{R}_{n}$. Let $j=i-1$ and $x=a^{2 j_{u}}$. Clearly, $x \neq 1(\bmod n)$ because of $i$ 's minimality. Moreover, $x \neq \equiv-1(\bmod n)$ since otherwise $a$ would belong to $\mathrm{R}_{n}$. Hence, $x \neq \equiv \pm 1$ $(\bmod n)$ but $x^{2} \equiv 1(\bmod n)$. Therefore, $\operatorname{gcd}(n, x+1)$ is a nontrivial factor of $n$.

(ii) Let $n=p_{1}^{m_{1}} p_{2}^{m_{2}} \cdots p_{r}^{m_{r}}$ be the decomposition of $n$ into prime factors. Let $p_{i}-1=2^{v_{i}} u_{i}$ for each $i$, where $u_{i}$ is odd. Let $\tau=\min \left\{v_{i} \mid 1 \leq i \leq r\right\}$. Monier has proved the following formulae [16]:

$$
\# \mathrm{~F}_{n}=\prod_{i=1}^{r} \operatorname{gcd}\left(n-1, p_{i}-1\right) \quad \text { and } \# \mathrm{R}_{n}=\left[1+\frac{2^{r \tau}-1}{2^{r}-1}\right] \prod_{i=1}^{r} \operatorname{gcd}\left(u, u_{i}\right) \text {. }
$$

(The formula for $\# F_{n}$ was discovered independently by Baillie and Wagstaff [4].) Moreover, $\tau \leq v[16]$, hence

$$
\operatorname{gcd}\left(n-1, p_{i}-1\right)=\operatorname{gcd}\left(2^{v} u, 2^{v_{i}} u_{i}\right)=2^{\min \left(v, v_{i}\right)} \operatorname{gcd}\left(u, u_{i}\right) \geq 2^{\tau} \operatorname{gcd}\left(u, u_{i}\right) .
$$

Therefore,

$$
\# \mathrm{~F}_{n} \geq 2^{r \tau} \prod_{i=1}^{r} \operatorname{gcd}\left(u, u_{i}\right)
$$


Finally, since $\tau \geq 1$ because $n$ is odd and $r \geq 2$ because $n$ is not a prime power,

$$
\begin{aligned}
\frac{\# \mathrm{~F}_{n}}{\# \mathrm{R}_{n}} & \geq \frac{2^{r \tau}}{1+\left(2^{r \tau}-1\right) /\left(2^{r}-1\right)}=\frac{2^{r}-1}{2^{1-r \tau}\left(2^{r-1}-1\right)+1} \\
& \geq \frac{2^{r}-1}{2^{1-r}\left(2^{r-1}-1\right)+1}=\frac{2^{r}-1}{2-2^{1-r}}=2^{r-1} \geq 2 .
\end{aligned}
$$

This proves that \# $\mathrm{F}_{n} \geq 2 \times \# \mathrm{R}_{n}$, hence \# $\mathrm{H}_{n}=\# \mathrm{~F}_{n}-\# \mathrm{R}_{n} \geq \# \mathrm{R}_{n}$.

Notice that a more exact formula is not much harder to establish:

$$
2^{r+x-1} \leq \# \mathrm{~F}_{n} / \# \mathrm{R}_{n} \leq 2^{x}\left(2^{r}-1\right)<2^{r+x}, \quad \text { where } \quad x=\sum_{i=1}^{r}\left[\min \left(v, v_{i}\right)-\tau\right] \geq 0 .
$$

In particular, $\mathrm{F}_{n}=\mathrm{R}_{n}$ when $n$ is a prime power $(r=1$ and $x=0)$.

There are several ways in which the notion of a handle can be generalized. It is clear from part (i) of Theorem 1 that any Fermat false witness that is however not a Rabin false witness can be used to split $n$ efficiently, but other numbers can be used as well. Let us define an $n$-splitter to be an integer $x$ such that $1<\operatorname{gcd}(x, n)<n$. Obviously, knowledge of any $n$-splitter can be used to compute a nontrivial factor of $n$ efficiently (using Euclid's algorithm), and there are exactly $n-\varphi(n)-1$ of them between 1 and $n$. What else could be used as handle?

As usual, let $n$ be an odd composite integer, and let $v$ and $u$ be integers such that $n-1=2^{v} u$, where $u$ is odd. The following definitions for handles are natural in the sense that they mimic the Rabin and Fermat false witnesses, respectively:

(i) $a$ is a Rabin handle $\left(a \in \mathrm{RH}_{n}\right)$ if $1 \leq a<n$ and

- $a^{u}-1$ is an $n$-splitter, or

- $a^{2 j_{u}}+1$ is an $n$-splitter for some $0 \leq j<v$.

(ii) $a$ is a Fermat handle $\left(a \in \mathrm{FH}_{n}\right)$ if $1 \leq a<n$ and - $a^{n-1}-1$ is an $n$-splitter.

We know from Theorem 1 that $H_{n} \subseteq R H_{n}$, where $H_{n}$ is the set of handles previously discussed (the Fermat false witnesses that are not Rabin false witnesses), but how many additional handles for splitting $n$ did we miss by concentrating only on $\mathrm{H}_{n}$ ? The following theorem tells us that we missed precisely the Fermat handles, and that there are at least $n-\varphi(n)-1$ of them.

Theorem 2. Let $n$ be an odd integer, $n>1$, and let $p_{1}^{m_{1}} p_{2}^{m_{2}} \cdots p_{r}^{m_{r}}$ be its decomposition into prime factors:

(i) $\mathrm{RH}_{n}=\mathrm{H}_{n} \cup \mathrm{FH}_{n}$,

(ii) $\mathrm{H}_{n} \cap \mathrm{FH}_{n}=\varnothing$,

(iii) $\# \mathrm{FH}_{n}=n-\# \mathrm{~F}_{n}-n \prod_{i=1}^{r}\left(1-\operatorname{gcd}\left(p_{i}-1, n-1\right) / p_{i}\right) \geq n-\varphi(n)-1$, and

(iv) $\# \mathrm{RH}_{n}=n-\# \mathrm{R}_{n}-n \prod_{i=1}^{r}\left(1-\operatorname{gcd}\left(p_{i}-1, n-1\right) / p_{i}\right)$.

(Part (iii) holds as well if $n$ is even, provided that the definition of $\mathrm{FH}_{n}$ is extended in the natural way to even numbers.) 
In order to simplify the proof, let us first state some useful facts whose justifications are left to the reader:

(a) In general, for any integers $x \geq 2$ and $k \geq 1, x^{k}-1$ is divisible by $x-1$; it is also divisible by $x+1$ provided $k$ is even. In particular, for any integers $a \geq 2$, $i \geq j \geq 0$ and $u \geq 1, a^{2^{i_{u}}}-1$ is divisible by $a^{2 j_{u}}-1$; it is also divisible by $a^{2 j_{u}}+1$ provided $j<i$.

(b) For any integers $a \geq 2, u \geq 1$, and any odd prime $p$, at most one member of $\left\{a^{u}-1\right\} \cup\left\{a^{2^{j_{u}}}+1 \mid j \geq 0\right\}$ is divisible by $p$. Indeed, if $p$ divides two members of this set, then the larger one is $a^{2 j_{u}}+1$ for some $j \geq 0$, whereas the product of all the smaller members of this set is $a^{2 j_{u}}-1$. But $p$ must also divide this product, hence $p=2$ for a contradiction.

(c) It is obvious that $\mathrm{R}_{n} \subseteq \mathrm{F}_{n}, \mathrm{R}_{n} \cap \mathrm{H}_{n}=\varnothing, \mathrm{F}_{n} \cap \mathrm{FH}_{n}=\varnothing$, and $\mathrm{R}_{n} \cap \mathrm{FH}_{n}=\varnothing$; it is somewhat less obvious, but it follows from fact (b), that $\mathbf{R}_{n} \cap \mathbf{R H}_{n}=\varnothing$.

(d) if $p$ is prime, then

$$
\#\left\{a \mid 0 \leq a<p \text { and } a^{n-1} \equiv 1(\bmod p)\right\}=\operatorname{gcd}(p-1, n-1) .
$$

(e) Consider any real number $c \geq 1$. It follows from elementary calculus that $\max \{x+c / x \mid 1 \leq x \leq c\}=1+c$.

Proof of Theorem 2. As always, let $v$ and $u$ be integers such that $n-1=2^{v} u$, where $u$ is odd.

(i) Consider any $a \in \mathrm{RH}_{n}$. By fact (a), $a^{n-1}-1$ is divisible by $a^{u}-1$ and by $a^{2 j_{u}}+1$ for each $j<v$. Thus, by definition of $\mathrm{RH}_{n}, \operatorname{gcd}\left(a^{n-1}-1, n\right)>1$. If this gcd is equal to $n$, then $a \in \mathrm{F}_{n}$ by definition. But $a \notin \mathrm{R}_{n}$ by fact (c). Therefore, $a \in \mathrm{F}_{n} \backslash \mathbf{R}_{n}=\mathbf{H}_{n}$. On the other hand, if this gcd is strictly between 1 and $n$ then $a \in \mathrm{FH}_{n}$ by definition. This shows that $\mathrm{RH}_{n} \subseteq \mathrm{H}_{n} \cup \mathrm{FH}_{n}$.

Now, consider any $a \in \mathrm{H}_{n} \cup \mathrm{FH}_{n}$. By fact (c), $a \notin \mathrm{R}_{n}$. By definition, $\operatorname{gcd}\left(a^{n-1}-1, n\right)>1$. Let $p$ be a prime factor of this gcd. Clearly, $a^{n-1} \equiv 1(\bmod p)$, which implies (because $p$ is a prime) that either $a^{u} \equiv 1(\bmod p)$ or $a^{2 j_{u}} \equiv-1(\bmod p)$ for some $j<v$. Thus either $\operatorname{gcd}\left(a^{u}-1, n\right)>1$ or $\operatorname{gcd}\left(a^{2^{j_{u}}}+1, n\right)>1$ for some $j<v$. But none of these gcd's can be $n$ since $a \notin \mathbf{R}_{n}$. Thus $a \in \mathbf{R H}_{n}$, which completes the proof that $\mathrm{H}_{n} \cup \mathrm{FH}_{n} \subseteq \mathrm{RH}_{n}$.

(ii) Immediate from the facts that $\mathrm{F}_{n} \cap \mathrm{FH}_{n}=\varnothing$ and $\mathrm{H}_{n} \subseteq \mathrm{F}_{n}$.

(iii) Consider any $0 \leq a<n$. It is clear that exactly one of the following three possibilities holds: $a \in \mathrm{F}_{n}, a \in \mathrm{FH}_{n}$, or $\operatorname{gcd}\left(a^{n-1}-1, n\right)=1$. Therefore, the equality in part (iii) will follow immediately after we show that

$$
\#\left\{a \mid 0 \leq a<n \text { and } \operatorname{gcd}\left(a^{n-1}-1, n\right)=1\right\}=n \prod_{i=1}^{r}\left(1-\frac{\operatorname{gcd}\left(p_{i}-1, n-1\right)}{p_{i}}\right) .
$$

To see this, consider any $i, 1 \leq i \leq r$. By fact (d),

$$
\#\left\{a \mid 0 \leq a<p_{i} \text { and } a^{n-1} \equiv 1\left(\bmod p_{i}\right)\right\}=\operatorname{gcd}\left(p_{i}-1, n-1\right) .
$$

Therefore,

$$
\#\left\{a \mid 0 \leq a<p_{i}^{m_{i}} \text { and } a^{n-1} \equiv 1\left(\bmod p_{i}\right)\right\}=p_{i}^{m_{i}-1} \operatorname{gcd}\left(p_{i}-1, n-1\right) .
$$


We thus conclude that

$$
\#\left\{a \mid 0 \leq a<p_{i}^{m_{i}} \text { and } a^{n-1} \not \equiv 1\left(\bmod p_{i}\right)\right\}=p_{i}^{m_{i}}-p_{i}^{m_{i}-1} \operatorname{gcd}\left(p_{i}-1, n-1\right) .
$$

From the Chinese Remainder Theorem, it follows that the number of $a$ such that $0 \leq a<n$ and $a^{n-1} \not \equiv 1\left(\bmod p_{i}\right)$ for each $1 \leq i \leq r$ is given by

$$
\prod_{i=1}^{r}\left(p_{i}^{m_{i}}-p_{i}^{m_{i}-1} \operatorname{gcd}\left(p_{i}-1, n-1\right)\right)=n \prod_{i=1}^{r}\left(1-\frac{\operatorname{gcd}\left(p_{i}-1, n-1\right)}{p_{i}}\right) .
$$

Thus the equality in part (iii) holds since $a^{n-1} \not \equiv 1\left(\bmod p_{i}\right)$ for each $1 \leq i \leq r$ precisely when $\operatorname{gcd}\left(a^{n-1}-1, n\right)=1$.

In order to show the inequality in part (iii) of the theorem, let $x_{i}$ denote $\operatorname{gcd}\left(p_{i}-1, n-1\right)$, let $\alpha(n)$ denote $\prod_{i=1}^{r} p_{i}$, the largest square-free divisor of $n$, and let $\beta(n)=\varphi(\alpha(n))$ denote $\prod_{i=1}\left(p_{i}-1\right)$. By the above cited formula of Monier and Baillie-Wagstaff, $\# \mathrm{~F}_{n}=\prod_{i=1}^{r} x_{i}$. Therefore, the equality we just proved implies that

$$
\begin{aligned}
\# \mathrm{FH}_{n} & =n-\prod_{i=1}^{r} x_{i}-n \prod_{i=1}^{r}\left(1-x_{i} / p_{i}\right) \\
& =n-\prod_{i=1}^{r} x_{i}-\frac{n}{\alpha(n)} \prod_{i=1}^{r}\left(p_{i}-x_{i}\right) .
\end{aligned}
$$

Consider now the function $\Xi_{n}$ in $r$ variables defined by

$$
\Xi_{n}\left(y_{1}, y_{2}, \ldots, y_{r}\right)=\prod_{i=1}^{r} y_{i}+\frac{n}{\alpha(n)} \prod_{i=1}^{r}\left(p_{i}-y_{i}\right),
$$

which is defined precisely so that \# $\mathrm{FH}_{n}=n-\Xi_{n}\left(x_{1}, x_{2}, \ldots, x_{r}\right)$. Because $1 \leq x_{i} \leq$ $p_{i}-1$ for each $1 \leq i \leq r$, it is clear that

$$
\# \mathrm{FH}_{n} \geq n-\max \left\{\Xi_{n}\left(y_{1}, y_{2}, \ldots, y_{r}\right) \mid 1 \leq y_{i} \leq p_{i}-1 \text { for each } 1 \leq i \leq r\right\} \text {. }
$$

In order to maximize the function $\Xi_{n}$, first notice that it is linear in each variable. Therefore, its maximum value occurs at a point where each $y_{i}$ is either 1 or $p_{i}-1$. Let $S$ be an arbitrary subset of $\{1,2, \ldots, r\}$ and let $x$ denote $\prod_{i \in S}\left(p_{i}-1\right)$. Notice that $\prod_{i \notin s}\left(p_{i}-1\right)=\beta(n) / x$ and $n \beta(n) / \alpha(n)=\varphi(n)$. Setting $y_{i}=p_{i}-1$ for $i \in S$ and $y_{i}=1$ for $i \notin S$, we thus have $\Xi_{n}\left(y_{1}, y_{2}, \ldots, y_{r}\right)=x+\varphi(n) / x$. Using fact (e), since $x$ can only take values between 1 and $\beta(n) \leq \varphi(n)$, we conclude that the value of $\Xi_{n}$ in the range of interest never exceeds $1+\varphi(n)$. We have thus established that $\# \mathrm{FH}_{n} \geq n-\varphi(n)-1$, as claimed.

(iv) Immediate from the first three parts of this theorem and from the obvious fact that $\# \mathrm{H}_{n}=\# \mathrm{~F}_{n}-\# \mathrm{R}_{n}$.

\section{How Good Is Rabin's Test?}

We must first ask the following question: what is Rabin's test good for? At least two answers come to mind: to decide if a given number is probably prime and to generate one or several random integers that are probably prime. We consider these two settings in turn, starting with the second. 


\subsection{How To Generate Random Numbers That Are Probably Prime}

The generation of large primes drawn with a uniform distribution from the set of all primes of a given size is of crucial importance in cryptography [21]. Although it is possible to generate such primes with certainty using the algorithms of [2], [1], their running time is currently too high to be used in practice. It is also possible to generate large certified primes efficiently by a variation on Pratt's nondeterministic algorithm [19] (generate the NP certificate and the resulting prime hand in hand) or by more sophisticated techniques [8], but the resulting distribution would not be uniform. Again, the most attractive solution in practice is to use Rabin's test as follows:

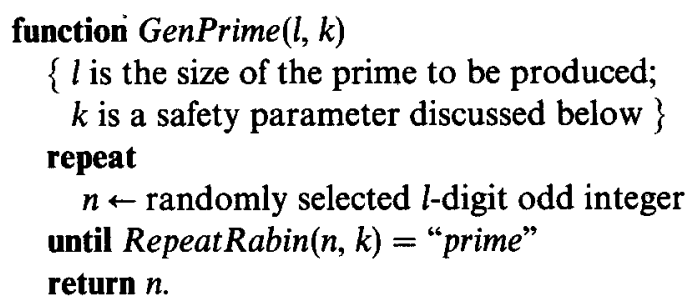

The resulting output is a probabilistic prime in the sense that we are not assured that it is indeed prime using the above algorithm, no matter how large we choose $k$. We can nonetheless increase our confidence in the number's primality by increasing the safety parameter $k$. (What a shame that Rabin's algorithm can certify those cryptographically useless composite numbers whereas it can only give probabilistic information on the useful primes! - which is precisely why the algorithms in [11] and [1] are of such (as yet theoretical) interest.)

In order to use GenPrime for cryptographic purposes, it is important that its probability of returning a composite integer be estimated. The popular belief is that

$$
\operatorname{prob}[\text { GenPrime }(l, k) \text { is composite }] \leq 4^{-k}
$$

because each of the $k$ rounds of RepeatRabin has a probability smaller than $1 / 4$ of failing on any given composite number. If we repeatedly use GenPrime to produce $m$ distinct "primes," we therefore expect on the average that at most $m \times 4^{-k}$ of them will turn out to be composite. For instance, Knuth writes [13, p. 379]:

If we certified a billion different primes with such a procedure, ${ }^{4}$ the expected number of mistakes would be less than $1 / 1,000,000$.

This assertion is true, but its proof is not so obvious. In particular, the reasoning given above is fallacious. Indeed, it is only true because prob [Rabin $(n)=$ "prime"] is much smaller than $1 / 4$ on most composite numbers. Should the error probability of Rabin's test be exactly $1 / 4$ on each and every composite odd integer, the

\footnotetext{
${ }^{4}$ Knuth does not explicitly say how he would use Rabin's test to certify those billion primes, except that he would run it " 25 -times-in-a-row" on each of them. It is our interpretation that he meant something along the lines of RepeatRabin $(\cdot, 25)$. Of course, Knuth's assertion is vacuously true if taken literally: if the integers thus certified are indeed "one billion primes", no mistakes are possible at all!
} 
number of expected errors in Knuth's quote could be significantly larger than $10^{9} \times 4^{-25} \approx 10^{-6}$.

Let $X$ stand for " $n$ is composite" and $Y_{k}$ for "Repeat Rabin $(n, k)$ returned "prime"." The basic theorem about Rabin's test obviously implies that $\operatorname{prob}\left[Y_{k} \mid X\right] \leq 4^{-k}$. However, this is not the probability relevant to the procedure GenPrime: rather, we are interested in $\operatorname{prob}\left[X \mid Y_{k}\right]$. This latter probability cannot be estimated (or even bounded away from 1) without specification of the probability distribution over $n$. For the moment, let $S$ be any set of odd integers and let $n$ be chosen randomly and uniformly in $S$. Let $p$ stand for the probability that $n$ be prime (i.e., $p=\#\{n \in S \mid n$ is prime $\} / \# S$ ), and let us assume that $0<p<1$. Elementary probability theory yields:

$$
\begin{aligned}
\operatorname{prob}\left[X \mid Y_{k}\right] & =\frac{\operatorname{prob}[X] \times \operatorname{prob}\left[Y_{k} \mid X\right]}{\operatorname{prob}\left[Y_{k}\right]} \\
& =\frac{\operatorname{prob}[X] \times \operatorname{prob}\left[Y_{k} \mid X\right]}{\operatorname{prob}[X] \times \operatorname{prob}\left[Y_{k} \mid X\right]+\operatorname{prob}[\operatorname{not} X] \times \operatorname{prob}\left[Y_{k} \mid \operatorname{not} X\right]} \\
& =\frac{\operatorname{prob}\left[Y_{k} \mid X\right]}{\operatorname{prob}\left[Y_{k} \mid X\right]+\frac{\operatorname{prob}[\operatorname{not} X] \times \operatorname{prob}\left[Y_{k} \mid \operatorname{not} X\right]}{\operatorname{prob}[X]}} \\
& =\frac{\operatorname{prob}\left[Y_{k} \mid X\right]}{\operatorname{prob}\left[Y_{k} \mid X\right]+p /(1-p)} \\
& \leq p^{-1} \times \operatorname{prob}\left[Y_{k} \mid X\right] .
\end{aligned}
$$

In particular, if prob $\left[Y_{k} \mid X\right] \ll p \ll 1$, we get that $\operatorname{prob}\left[X \mid Y_{k}\right]$ is almost equal to $p^{-1} \times \operatorname{prob}\left[Y_{k} \mid X\right]$. On the other hand, if $p \ll \operatorname{prob}\left[Y_{k} \mid X\right]$, then the above calculation shows that prob $\left[X \mid Y_{k}\right]$ is very close to 1 , which means that $n$ is almost certainly composite whenever Rabin's test finds it probably prime $k$ times in a row! It is thus clear that $\operatorname{prob}\left[X \mid Y_{k}\right] \leq 4^{-k}$ cannot be a direct consequence of the mere fact that $\operatorname{prob}\left[Y_{k} \mid X\right] \leq 4^{-k}$. Worse still, prob $\left[X \mid Y_{k}\right] \leq 4^{-k}$ might even be false. This line of thought is carried out to the extreme in [5] and unduly pessimistic consequences are drawn. Nonetheless, the more detailed analysis below shows that the precautions advocated in [5] are not necessary. In particular, the probability of GenPrime $(l, k)$ returning a composite integer actually decreases as the value of $l$ increases (for any fixed value of $k$ ).

In order to bound prob $\left[X \mid Y_{k}\right]$ more precisely, a tighter bound on prob $\left[Y_{k} \mid X\right]$ is needed. Thus, we must study the average behavior of Rabin's test, rather than its much simpler worst case. This question was raised and solved to a large degree by Erdös and Pomerance [9], but only for the case of a single iteration of Rabin's test, corresponding to $k=1$ : the average number of false witnesses for integers up to $n$ does not exceed $n / L(n)^{\xi(n)}$, where $L(n)$ is defined as $n^{\ln \ln \ln n / \ln \ln n}$ and $\xi: \mathbb{N} \rightarrow \mathbb{R}$ is a function such that $\lim _{n \rightarrow \infty} \xi(n)=1$ (this holds even for the Fermat congruence). One may assume without loss of generality that $L(n)^{\xi(n)}$ is monotone increasing.

However, successive runs of Rabin's test on a given randomly chosen odd integer $n$ are not independent in the sense that if a first run finds that $a_{1} \in \mathbf{R}_{n}$, this increases 
very significantly the likelihood that the next run will also find that $a_{2} \in \mathrm{R}_{n}$, where $a_{1}$ and $a_{2}$ are randomly, uniformly, and independently chosen between 2 and $n-2$. (Let us disregard here the fact that the second run would not even take place should the first run find that $a_{1} \notin \mathrm{R}_{n}$.) Consult [18] for numerical evidence about this. This lack of independence prevents us from asserting that prob $\left[Y_{k} \mid X\right]=\left(\operatorname{prob}\left[Y_{1} \mid X\right]\right)^{k}$. Nonetheless, the basic fact that $\operatorname{prob}\left[a_{2} \in \mathrm{R}_{n}\right] \leq 1 / 4$ remains true as long as $n$ is composite, and this applies equally well to each and every run, independently of what happens in the other runs. Therefore,

$$
\operatorname{prob}\left[Y_{k} \mid X\right] \leq \frac{\operatorname{prob}\left[Y_{1} \mid X\right]}{4^{k-1}} .
$$

Let us now be more specific about the set $S$ of odd integers among which $n$ is randomly and uniformly drawn, so that our study corresponds to a call on GenPrime $(l, k)$ for positive integers $l$ and $k$. Let $S$ be the set of $l$-digit odd integers. The prime number theorem [12] tells us that

$$
p=\operatorname{prob}[n \text { is prime } \mid n \in S] \approx \beta / l,
$$

where $\beta=\log _{10} e^{2} \approx 0.87$ (see Lemma 1 in [5]). Moreover, the study in [9] tells us that

$$
\operatorname{prob}\left[Y_{1} \mid X\right] \leq 1 / P\left(10^{l-1}\right),
$$

where $P(n)$ stands for $L(n)^{\xi(n)}$. Hence,

$\operatorname{prob}[$ GenPrime $(l, k)$ is composite $]=\operatorname{prob}\left[X \mid Y_{k}\right]$

$$
\leq p^{-1} \times \operatorname{prob}\left[Y_{k} \mid X\right] \leq \frac{4}{\beta} \frac{l}{P\left(10^{l-1}\right)} 4^{-k} .
$$

But clearly $\lim _{l \rightarrow \infty} l / P\left(10^{l-1}\right)=0$, thus prob[GenPrime $(l, k)$ is composite $] \leq 4^{-k}$ for all sufficiently large $l$, which proves Knuth's previously quoted claim (at least if the numbers considered are sufficiently large).

Let us stress again that, although one's confidence in the primality of the output of GenPrime $(l, k)$ increases with the value of $k, l / P\left(10^{l-1}\right)$ is already so small for large values of $l$ that an arbitrarily low error probability can be obtained with a mere call on GenPrime $(l, 1)$ if large enough primes are sought. In fact, this remains true even if the simpler Fermat congruence is used instead of Rabin's test, but one should resist the temptation of doing this because Fermat's congruence is only simpler conceptually but never faster (and often slower) to compute.

\subsection{How To Decide on the Primality of a Given Integer}

Suppose some odd integer $\boldsymbol{n}$ is given to you. You are to decide whether you think it is prime or not. You therefore run Rabin's test for some number $k$ of rounds, and it never finds $n$ to be composite. What can you tell from this?

One obviously wrong answer is: "this number is prime with probability $1-4^{-k}$." This makes no sense because any given integer is either prime or not.

The classic answer is: "I believe this number to be prime, and my error probability 
is at most $4^{-k}$ (in the sense that I expect to be wrong at most once every $4^{k}$ such statements if you quiz me long enough)." This is wrong as well because no estimate on the error probability of "I believe this number to be prime" can be made without an a priori estimate on the probability that the number is prime. If you know that $n$ was chosen randomly and uniformly among the odd integers of some given size, Section 3.1 is relevant. However, if you do not know where the number comes from, you are at a complete loss.

Still, there is one thing you can say: "I believe this number to be prime, and if I am wrong I have observed a natural phenomenon whose probability of occurrence was bounded by $4^{-k}$." This appears to be the strongest statement one can infer in general from running Rabin's test $k$ times on a given integer that is not thus found to be composite.

As mentioned in the abstract, this observation is not restricted to primality testing. Whenever one runs any probabilistic algorithm that is not Las Vegas, care must be taken as to how to interpret the outcome. This general issue is discussed in [6].

\section{Open Problems}

It would be interesting to analyse the average and normal behavior of \# $\mathrm{FH}_{n}$ and $\# \mathrm{RH}_{n}$, much like the analyses of $\# \mathrm{~F}_{n}$ and $\# \mathrm{R}_{n}$ found in [9]. It would also be interesting to analyse more tightly the probability that RepeatRabin $(n, k)=$ "prime" when $n$ is a composite integer randomly and uniformly chosen among all $l$-digit composite integers; in particular, where does it lie between $P^{k}$ and $P / 4^{k-1}$, where $P$ stands for the probability corresponding to $k=1$ ? (We conjecture that it is much closer to $P / 4^{k-1}$ than to $P^{k}$, i.e., that our analysis was not too pessimistic.) Finally, a more precise analysis of how and how fast the function $\xi(n)$ converges to 1 (a question left unresolved in [9]) would be crucial in establishing from which values of $l$ it is true that, for every integer $k \geq 1$, prob $[\operatorname{GenPrime}(l, k)$ is composite $] \leq 4^{-k}$.

\section{Acknowledgments}

The first observation sprung from an idea attributed to Silvio Micali by Charles $\mathrm{H}$. Bennett (private communication) to the effect that any algorithm for extracting square roots modulo a prime can be turned into a probabilistic algorithm to decide primality. However, this origin is no longer perceptible in this paper (but see [5]). The proof of Theorem 1(ii) given here was suggested to one of the authors by Evangelos Kranakis; it is much nicer but far less elementary than our original proof. A very simple proof of Theorem 1 for the case when $n \equiv 3(\bmod 4)$ can be found in [5].

We wish to thank all those who showed interest for this work at the Crypto 86 meeting, in particular, Yvo Desmedt, Oded Goldreich, Gus Simmons, and Moti Yung. An ideal working environment was provided by David Chaum to one of the authors for the completion of this work. Finally, we are very grateful to Ernest F. Brickell, the Journal's editor-in-chief, for his efficiency in handling our paper. 


\section{References}

[1] Adleman, L., and M.-D. Huang, Recognizing primes in random polynomial time, Proceedings of the 19th Annual ACM Symposium on the Theory of Computing, pp. 462-469, 1987.

[2] Adleman, L., C. Pomerance, and R. Rumely, On distinguishing prime numbers from composite numbers, Annals of Mathematics, vol. 117, pp. 173-206, 1983.

[3] Babai, L., Monte Carlo algorithms in graph isomorphism testing, Rapport de Recherches du Département de Mathématiques et de Statistiques, D.M.S. \# 79-10, Université de Montréal, 1979.

[4] Baillie, R., and S. S. Wagstaff, Jr., Lucas pseudoprimes, Mathematics of Computation, vol. 35, no. 152, pp. 1392-1417, 1980.

[5] Beauchemin, P., G. Brassard, C. Crépeau, and C. Goutier, Two observations on probabilistic primality testing, Advances in Cryptology-Crypto 86 Proceedings, Springer-Verlag, New York, pp. 443-450, 1987.

[6] Brassard, G., and P. Bratley, Algorithmics: Theory and Practice, Prentice-Hall, Englewood Cliffs, New Jersey, 1988.

[7] Cohen, H., and A. K. Lenstra, Implementation of a new primality test, Mathematics of Computation, vol. 48 , no. 177 , pp. 103-121, 1987.

[8] Couvreur, C., and J.-J. Quisquater, An introduction to fast generation of large prime numbers, Philips Journal of Research, vol. 37, nos. 5/6, pp. 231-264, 1982.

[9] Erdös, P., and C. Pomerance, On the number of false witnesses for a composite number, Mathematics of Computation, vol. 46, no. 173, pp. 259-279, 1986.

[10] Gill, J., Computational complexity of probabilistic Turing machines, SI AM Journal on Computing, vol. 6, no. 4, pp. 675-695, 1977.

[11] Goldwasser, S., and J. Kilian, Almost all primes can be quickly certified, Proceedings of the 18th Annual ACM Symposium on the Theory of Computing, pp. 316-329, 1986.

[12] Hardy, G. H., and E. M. Wright, An Introduction to the Theory of Numbers, Fifth edition, Oxford Science Publications, 1979.

[13] Knuth, D. E., The Art of Computer Programming, Volume 2, Second edition, Addison-Wesley, Reading, Massachusetts, 1981.

[14] Kranakis, E., Primality and Cryptography, Wiley-Teubner Series in Computer Science, 1986.

[15] Miller, G. L., Riemann's hypothesis and tests for primality, Journal of Computer and System Sciences, vol. 13, pp. 300-317, 1976.

[16] Monier, L., Evaluation and comparison of two efficient probabilistic primality testing algorithms, Theoretical Computer Science, vol. 11, pp. 97-108, 1980.

[17] Pomerance, C., The search for prime numbers, Scientific American, vol. 247, no. 6, pp. 136-147, 1982.

[18] Pomerance, C., J. L. Selfridge, and S. S. Wagstaff, Jr., The pseudoprimes to $25.10^{9}$, Mathematics of Computation, vol. 35, no. 151, pp. 1003-1026, 1980.

[19] Pratt, V., Every prime has a succinct certificate, SIAM Journal on Computing, vol. 4, no. 3, pp. 214-220, 1975.

[20] Rabin, M. O., Probabilistic algorithm for testing primality, Journal of Number Theory, vol. 12, pp. $128-138,1980$.

[21] Rivest, R. L., A. Shamir, and L. Adleman, A method for obtaining digital signatures and publickey cryptosystems, Communications of the Association for Computing Machinery, vol. 21, no. 2, pp. $120-126,1978$.

[22] Solovay, R., and V. Strassen, A fast Monte Carlo test for primality, SIAM Journal on Computing, vol. 6, pp. 84-85, 1977; erratum in vol. 7, p. 118, 1978. 